\title{
Vigilancia Tecnológica de la Utilización de Criterios de Riesgo para la Gestión de Equipos Biomédicos
}

\author{
I. Ríos Cuartas ${ }^{\psi}$, B. Galeano Upegui, N. Escobar Mora \\ Grupo de Investigación en Bioingeniería, Línea de Ingeniería Clínica, Medellín, Colombia
}

\begin{abstract}
Resumen - El presente artículo expone una vigilancia tecnológica realizada para encontrar criterios de evaluación de riesgos usados para gestionar equipos biomédicos. Se presentan las ecuaciones de búsqueda planteadas con sus respectivos resultados y el análisis de acuerdo con la ecuación con la cual se hallaron los resultados de mayor relevancia. El análisis presentado corresponde a la dinámica de publicación en el tiempo, medios de publicación, autores relacionados, instituciones destacadas, países destacados, tipos de documento y áreas temáticas con el fin de identificar particularmente autores, países, instituciones y medios de publicación líderes en el tema. En la sección de resultados se analizan los artículos de los principales medios de publicación debido a la pertinencia de los enfoques según el marco regulatorio, paciente y dispositivos médicos que ofrecen. Con el análisis de resultados se obtuvo información concluyente que permitió observar la importancia de la generación de normativa que regule los dispositivos médicos durante su ciclo de vida apoyada en la gestión del riesgo, así como la conveniencia de realizar análisis de riesgos basado en evidencia.
\end{abstract}

Palabras clave - Criterio de evaluación del riesgo, gestión de equipos biomédicos, gestión del riesgo.

\section{Technological Surveillance for the Use of Risk Assessment Criteria used to Manage Biomedical Equipment}

\begin{abstract}
This article presents a technological surveillance proposal for risk assessment criteria used to manage biomedical equipment. We present the search equations, along with their respective results and the analysis according to the equation with which the most relevant results were found. The analysis presented corresponds to the dynamics of publications in time, means of publication, related authors, prominent institutions, highlighted countries, document types and thematic areas, in order to identify particular authors, countries, institutions and publishing media leaders on the subject. In the results section, the articles of the main means of publication are analyzed per the pertinence of approaches, according to the regulatory framework, and the patient and medical devices that they offer. From the analysis of results, we obtained conclusive information that enabled us to observe the importance of the generation of norms regulating medical devices during their life cycle, supported by risk management, as well as, the convenience of conducting evidence-based risk analysis.
\end{abstract}

Keywords-Assessment criteria, biomedical equipment management, risk management.

\% Dirección para correspondencia: isabel.rios@upb.edu.co

DOI: https://doi.org/10.24050/19099762.n21.2017.1174 


\section{VigilânCia Tecnológica da Utilização de Critérios de Risco para A Gestão de Equipamentos Biomédicos}

Resumo-O presente artigo expõe uma vigilância tecnológica realizada para encontrar critérios de avaliação de riscos usados para gerenciar equipamentos biomédicos. Apresentam-se as equações de busca propostas com seus respectivos resultados e a análise de acordo à equação com a qual acharam-se os resultados de maior relevância. A análise apresentada corresponde à dinâmica de publicação no tempo, meios de publicação, autores relacionados, instituições destacadas, países destacados, tipos de documento e áreas temáticas com o fim de identificar particularmente autores, países, instituições e meios de publicação líderes no tema. Na secção de resultados analisam-se os artigos dos principais meios de publicação devido à pertinência dos enfoques segundo o marco regulamentar, paciente e dispositivos médicos que oferecem. Com a análise de resultados obteve-se informação concludente que permitiu observar a importância da geração de regulamento que regule os dispositivos médicos durante seu ciclo de vida apoiada na gestão do risco, bem como a conveniência de realizar análise de riscos baseado em evidência.

Palavras-chave-Critério de avaliação do risco, gestão de equipamentos biomédicos, gestão do risco.

\section{INTRODUCCIÓN}

$\mathrm{E}^{\prime}$ objetivo de este trabajo de investigación es encontrar los criterios de evaluación del riesgo que se utilizan en la actualidad para gestionar los equipos biomédicos, para ello la vigilancia tecnológica es la herramienta apropiada debido a que por medio de la recolección de información se encuentra el estado actual de un asunto particular ya que es una metodología sistematizada [1].

La metodología para la vigilancia tecnológica que se presenta en este artículo consta de los siguientes pasos: (1) planear identificando necesidades, (2) buscar y capturar información, (3) analizarla y estructurarla [2], debido a que los resultados del estudio serán la base para futuras investigaciones.

Con esta vigilancia tecnológica se busca contribuir a la seguridad de los usuarios de la tecnología de la salud gestionando los riesgos asociados a su uso para mitigar la ocurrencia de eventos e incidentes adversos en el proceso de atención [3]. Así como aportar a la gestión de los equipos biomédicos en la optimización de los recursos durante las diferentes etapas de su ciclo de vida, ya que la gerencia de activos es un proceso medular en las entidades que prestan servicios de salud [4].

\section{Metodología}

El método para la vigilancia tecnológica descrito en líneas anteriores tiene como primer paso la identificación de la necesidad, para el caso de estudio son las palabras apropiadas para establecer las ecuaciones de búsqueda de forma que se logren encontrar los criterios de evaluación del riesgo con los que se administra la tecnología sanitaria. Las palabras utilizadas para hacer las ecuaciones de la búsqueda son las siguientes:
- Risk management: Palabra que en español se traduce como Gestión del riesgo, la norma internacional ISO 31000:2009 provee los principios y directrices generales [5].

- Evaluation methods: Es el tesauro para criterio de evaluación [6].

- Risk assessment criteria: Su traducción es Criterio de evaluación del riesgo.

- Asset management: En español es Gestión de activos, la información general, principios y terminología se encuentra en la norma ISO 55000: 2014 [7].

- Medical equipment Management: Se traduce como Gestión de Equipos biomédicos, Medical

- Equipment es el tesauro para equipo sanitario [8].

- Medical device management: La traducción de esta expresión es Gestión de Dispositivos médicos.

- Health technology management: Este término se traduce como Gestión de tecnología de la salud.

Luego de tener las palabras para las ecuaciones se procede a realizar la búsqueda en la base de datos Scopus, debido a que es la herramienta preferente para realizar estudios bibliométricos, por su gran contenido y poseer analizador de resultados; además la literatura científica es examinada por especialistas y permite obtener un panorama multidisciplinario [9].

Las ecuaciones y sus correspondientes resultados se aprecian en la Tabla 1. En las ecuaciones el campo de búsqueda se hace por título del artículo, resumen y palabras clave, adicional a esto se buscaron publicaciones realizadas en los últimos 10 años, es decir a partir del 2007. 
Tabla 1. Ecuaciones de búsqueda para la vigilancia tecnológica

\begin{tabular}{clc}
\hline$N^{\circ}$ & \multicolumn{1}{c}{ Ecuación de búsqueda } & Resultados \\
\hline 1 & $\begin{array}{l}\text { Risk management and evaluation methods and } \\
\text { asset management }\end{array}$ & 223 \\
2 & $\begin{array}{l}\text { Risk management and evaluation methods and } \\
\text { medical device management }\end{array}$ & 263 \\
3 & $\begin{array}{l}\text { Risk management and evaluation methods and } \\
\text { medical equipment management }\end{array}$ & 141 \\
4 & $\begin{array}{l}\text { Risk management and evaluation methods and } \\
\text { health technology management }\end{array}$ & 356 \\
5 & $\begin{array}{l}\text { Risk assessment criteria and asset management } \\
6\end{array}$ & $\begin{array}{l}\text { Risk assessment criteria and medical device } \\
\text { management }\end{array}$ \\
$6 *$ & $\begin{array}{l}\text { Risk assessment criteria and “medical device” } \\
\text { management * }\end{array}$ & $27 * 3$ \\
7 & $\begin{array}{l}\text { Risk assessment criteria and medical equipment } \\
\text { management }\end{array}$ & $\begin{array}{l}\text { Risk assessment criteria and health technology } \\
\text { management }\end{array}$ \\
\hline
\end{tabular}

De los resultados que se obtienen con cada ecuación se realiza la revisión de los documentos, la ecuación con la que se encontraron los archivos mejor destacados es "risk assessment criteria and "medical device" management" con la que se obtienen 27 resultados, los datos encontrados son los siguientes:

\section{A. Dinámica de publicación en el tiempo}

En esta categoría el año en el que más producciones escritas se realizaron con los temas criterio de evaluación del riesgo y gestión de dispositivos médicos fue en el año 2014 con un total de 9 publicaciones, por el contrario, en el 2011 no se registraron documentos, luego para los años 2012, 2013 y 2016 se publicaron 3 documentos por año y en 2015 se registra el segundo año con mayor cantidad de publicaciones con un total de 4, en la Fig. 1. se observa el gráfico correspondiente a los documentos por año de publicación [10].
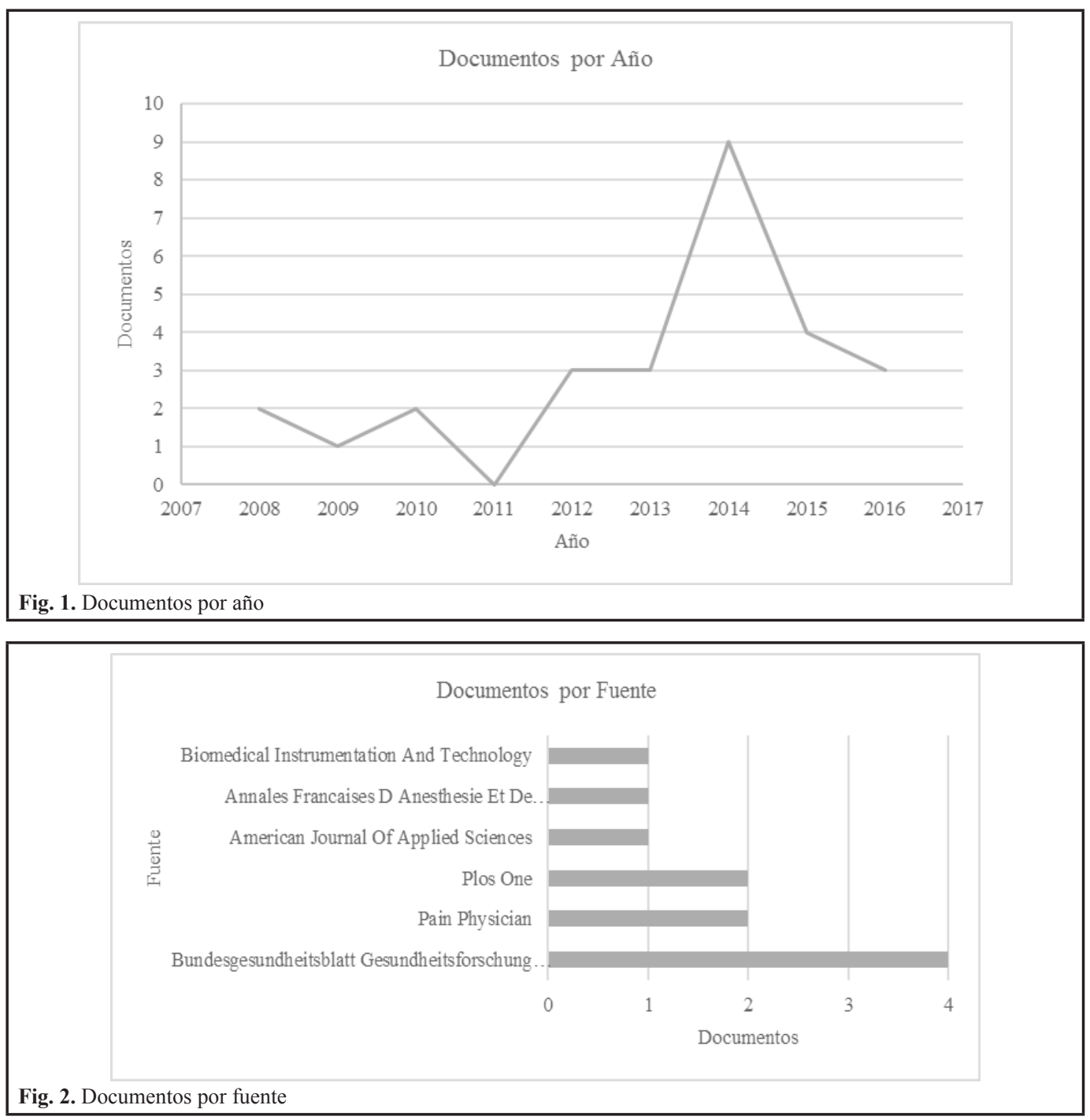


\section{B. Medios de Publicación}

En el análisis por fuente entre los años 2007 y 2017, Fig. 2., se observa que la revista Bundesgesundheitsblatt Gesundheitsforschung Gesundheitsschutz es la que tiene más publicaciones con un total de 4 en temas de dispositivos médicos y sus correspondientes evaluaciones del riesgo, las revistas Pain Physician y Plos One tiene cada una 2 divulgaciones [10].

\section{Autores relacionados}

La Fig. 3. muestra la cantidad de publicaciones por autor, se presentan los 10 escritores que mayor cantidad de publicaciones han realizado desde el 2007 hasta el presente sobre gestión de riesgos en dispositivos médicos. Entre los autores se destacan Benyamin, Boswell, Candido, Cohen, Diwany JanB, todos han realizado 2 publicaciones [10].

\section{Instituciones destacadas}

Este análisis presenta las instituciones que han estado en los últimos 10 años trabajando en temas de gestión del riesgo en tecnología de la salud, al igual que en el análisis por autor se observa que cada institución cuenta con 2 publicaciones, como se aprecia en el gráfico de la Fig. 4. Del grupo analizado se resalta el trabajo realizado por la Universidad de Louisville y Rheinisch-Westfalische Technische Hochschule Aachen, así como el centro del Millennium Pain Center [10].

\section{E. Países destacados}

Para el análisis por País se tienen 10 como los de mayor producción científica en las áreas particulares de búsqueda, en esta categoría el orden descendente por cantidad de publicaciones para los principales territorios es Estados Unidos con 8, Alemania con 7, Canadá con 5 y Reino Unido con 2; esto se visualiza en la Fig. 5. [10].
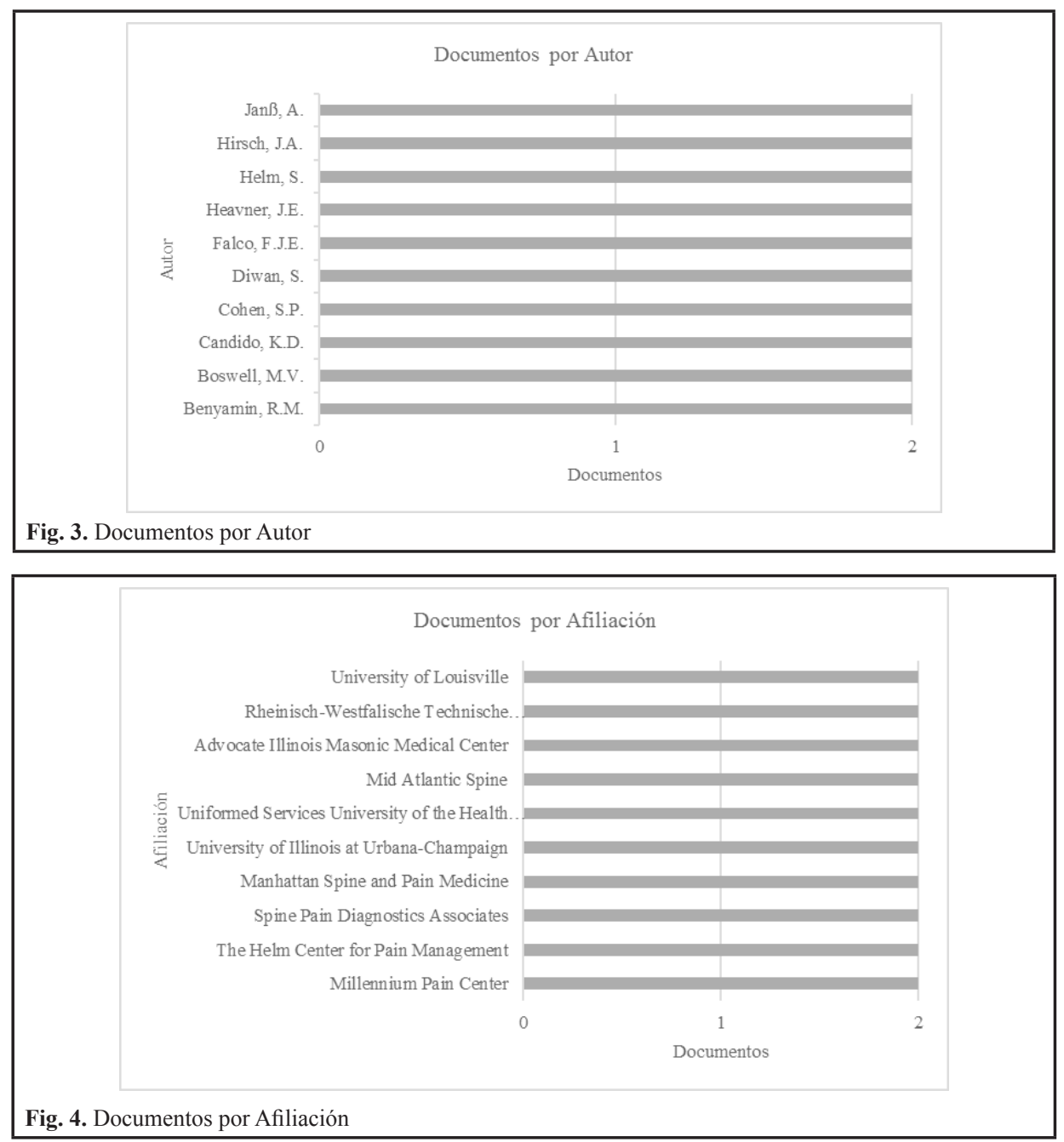
Ríos Cuartas I., Galeano Upegui B., Escobar Mora N. Criterios de Riesgo para la Gestión de Equipos Biomédicos

61

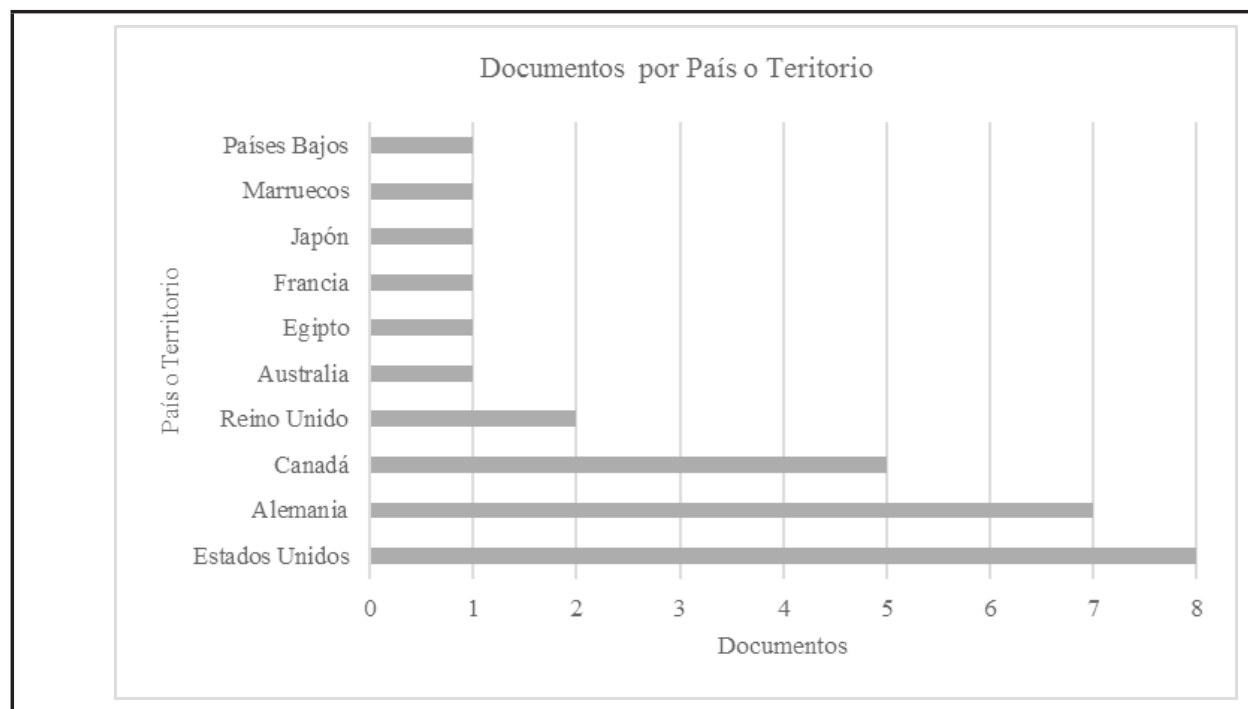

Fig. 5. Documentos por país o territorio

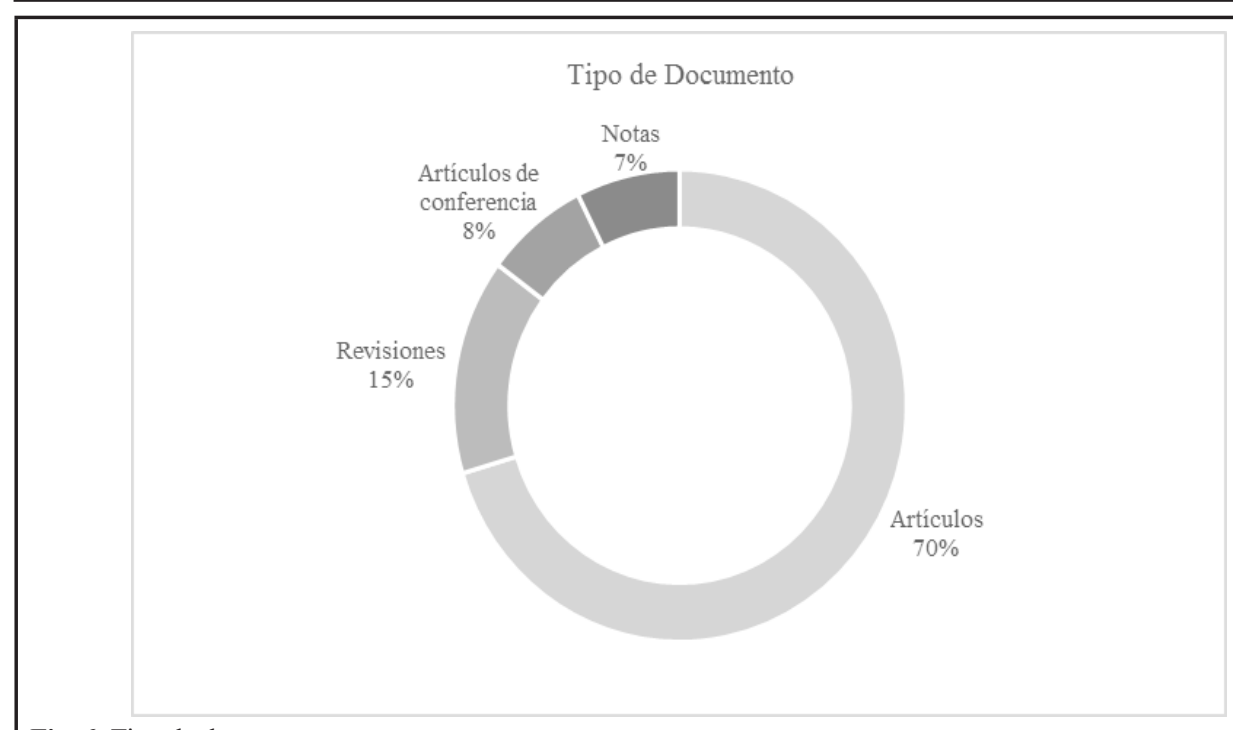

Fig. 6. Tipo de documento

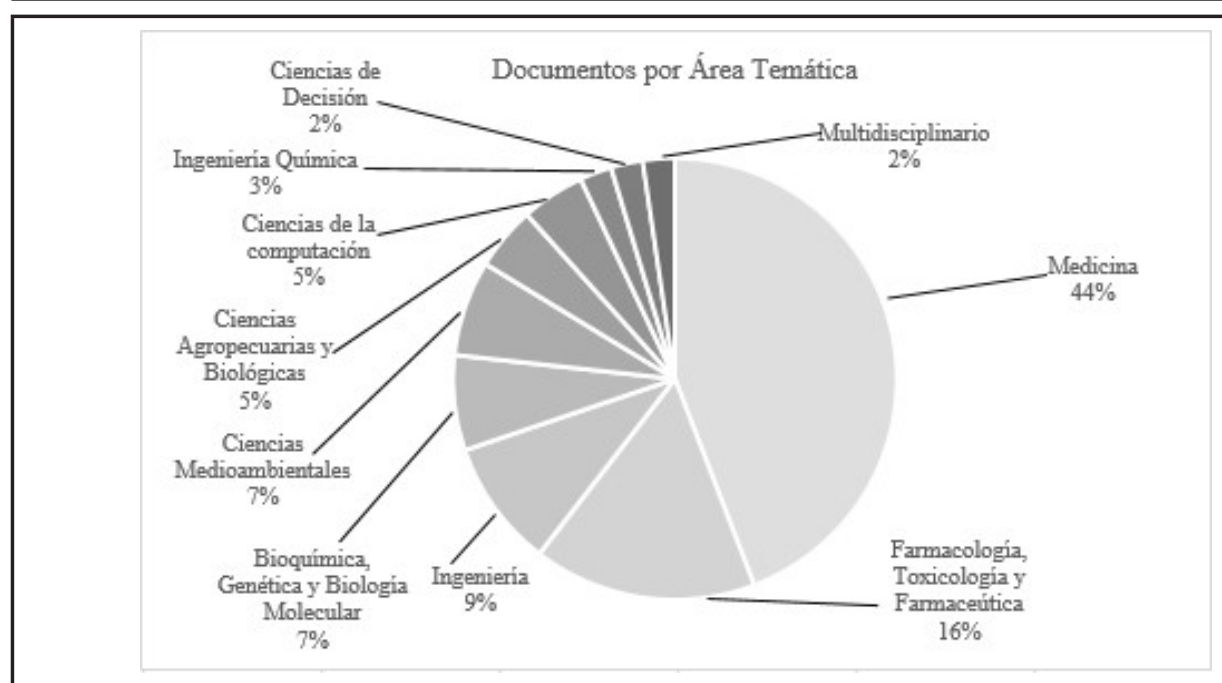

Fig. 7. Documentos por área temática 


\section{F. Tipos de documento}

El tipo de documento en el que se realiza la publicación para los 27 documentos analizados es en primer lugar Artículo con un $70 \%$, en segundo lugar, Revisiones con un $15 \%$, luego se encuentra con un $8 \%$ Artículos de conferencia y finalmente las Notas tienen un 7\%. De la Fig. 6. se observa que el medio de preferencia para la divulgación científica en los temas de gestión de dispositivos médicos y gestión del riesgo es el artículo [10].

\section{G. Áreas temáticas}

Las principales áreas temáticas a las que pertenecen las publicaciones resultantes con la ecuación de búsqueda son: Ciencias Medioambientales y Bioquímica, Genética y Biología Molecular con 7\%, Ingeniería con $9 \%$, Farmacología, Toxicología y Farmacéutica con 16\% y finalmente Medicina con 44\%, como se muestra en la Fig. 7. Con estos datos se observa que los temas analizados principalmente se estudian en áreas afines a la salud [10].

\section{Resultados}

La vigilancia tecnológica fue abordada metodológicamente desde 7 categorías diferentes, los resultados de esta se plantearán de acuerdo a los documentos encontrados por medio de publicación, se tendrán en cuenta las tres revistas con mayor cantidad de publicaciones por la pertinencia de sus contenidos.

\section{A. Bundesgesundheitsblatt Gesundheitsforschung Gesundheitsschutz}

Las publicaciones de esta revista se presentan en la Tabla 2, en el primer artículo se presenta el marco regulatorio en Alemania para los dispositivos médicos teniendo en cuenta la identificación, evaluación y mitigación de riesgos [10].

En la segunda publicación se hace referencia al marco regulatorio de Alemania para los ensayos clínicos en dispositivos médicos con clasificación del riesgo III y dispositivos implantables, así como la experiencia del Instituto Federal de Medicamentos y Dispositivos médicos en solicitudes y orientaciones a los usuarios. De la misma forma el tercer artículo muestra las regulaciones alemanas para fabricantes y productos sanitarios de alto riesgo [10].

El último artículo analizado de esta revista presenta el desarrollo de una metodología analítica y un software para hacer análisis prospectivo del riesgo humano para ayudar a los fabricantes de dispositivos médicos a identificar y controlar riesgos de forma eficiente en la interacción hombre- máquina [10].
Tabla 2. Documentos publicados por Bundesgesundheitsblatt Gesundheitsforschung Gesundheitsschutz

\begin{tabular}{|c|c|c|c|}
\hline $\mathrm{N}^{\circ}$ & Título & Autores & Año \\
\hline 1 & $\begin{array}{l}\text { Medical devices: Regulatory } \\
\text { framework and contribution of the } \\
\text { German Federal Institute for Drugs } \\
\text { and Medical Devices (BfArM) to } \\
\text { the safe application [Dispositivos } \\
\text { médicos: Marco reglamentario y } \\
\text { contribución del Instituto Federal } \\
\text { Alemán de Medicamentos y } \\
\text { Dispositivos Médicos (BfArM) para } \\
\text { la aplicación segura] }\end{array}$ & $\begin{array}{l}\text { Lauer W., } \\
\text { Stößlein, E., } \\
\text { Brinker, A., } \\
\text { Broich, K. }\end{array}$ & 2014 \\
\hline 2 & $\begin{array}{l}\text { Experiences and recommendations } \\
\text { of the GermanFederal Institute } \\
\text { for Drugs and Medical Devices } \\
\text { (BfArM) concerning clinical } \\
\text { investigation of medical devices } \\
\text { and the evaluation of serious } \\
\text { adverse events (SAE) [Experiencias } \\
\text { y recomendaciones del Instituto } \\
\text { Federal Alemán de Medicamentos } \\
\text { y Dispositivos Médicos (BfArM) } \\
\text { sobre la investigación clínica de } \\
\text { dispositivos médicos y la evaluación } \\
\text { de eventos adversos serios (SAE)] }\end{array}$ & $\begin{array}{l}\text { Renisch B., } \\
\text { Lauer, W., }\end{array}$ & 2014 \\
\hline 3 & $\begin{array}{l}\text { Focus Notified Bodies: New } \\
\text { requirements for designation and } \\
\text { monitoring [Foco en los organismos } \\
\text { notificados: nuevos requisitos para } \\
\text { la designación y el seguimiento] }\end{array}$ & $\begin{array}{l}\text { Poos, U., } \\
\text { Edelhäuser, R. }\end{array}$ & 2014 \\
\hline 4 & $\begin{array}{l}\text { Usability first: Model-based } \\
\text { approach for the use-oriented } \\
\text { risk analysis of medical devices } \\
\text { [Usabilidad primero: Enfoque } \\
\text { basado en modelos para el análisis } \\
\text { de riesgo orientado al uso de } \\
\text { dispositivos médicos] }\end{array}$ & $\begin{array}{l}\text { Janß, A., } \\
\text { Radermacher, } \\
\text { K. }\end{array}$ & 2014 \\
\hline
\end{tabular}

NOTA 1: Las traducciones de los títulos de los artículos son no oficiales.

\section{B. Pain Physician}

En la revista Pain Physician se analizan dos documentos en los que se divulga, en primer lugar, un instrumento para el manejo del dolor mediante la evaluación de calidad y evaluación de riesgos de Bias, se incluyen los criterios de revisión de Cochrane, este instrumento es superior $\mathrm{y}$ ofrece información amplia y específica. La segunda publicación que se analiza presenta el desarrollo de un instrumento específico para el manejo intervencionista del dolor, para este desarrollo se basan en criterios de revisión de Cochrane y se utilizan criterios de evaluación de riesgo y de calidad [10]. Lo anterior se aprecia en la Tabla 3. 
Tabla 3. Documentos publicados por Pain Physician

\begin{tabular}{|c|c|c|c|}
\hline $\mathrm{N}^{\circ}$ & Título & Autores & Año \\
\hline 1 & $\begin{array}{l}\text { Assessment of } \\
\text { methodologic quality } \\
\text { of randomized trials of } \\
\text { interventional techniques: } \\
\text { Development of an } \\
\text { interventional pain } \\
\text { management specific } \\
\text { instrument [Evaluación de } \\
\text { la calidad metodológica de } \\
\text { los ensayos aleatorios de } \\
\text { técnicas intervencionistas: } \\
\text { Desarrollo de un } \\
\text { instrumento específico } \\
\text { para el manejo del dolor } \\
\text { intervencionista] }\end{array}$ & $\begin{array}{l}\text { Manchikanti, } \\
\text { L., Hirsch, } \\
\text { J.A., Cohen, S.P., } \\
\text { (...), Racz, G.B., Raj, } \\
\text { Prithvi P. }\end{array}$ & 2014 \\
\hline 2 & $\begin{array}{l}\text { Development of } \\
\text { an interventional } \\
\text { pain management } \\
\text { specific instrument } \\
\text { for methodologic } \\
\text { quality assessment of } \\
\text { nonrandomized studies of } \\
\text { interventional techniques } \\
\text { [Desarrollo de un } \\
\text { instrumento específico } \\
\text { para la gestión del dolor } \\
\text { intervencionista para la } \\
\text { evaluación de la calidad } \\
\text { metodológica de estudios } \\
\text { no aleatorizados de } \\
\text { técnicas intervencionistas] }\end{array}$ & $\begin{array}{l}\text { Manchikanti, } \\
\text { L., Hirsch, } \\
\text { J.A., Heavner, J.E., } \\
\text { (...), Racz, } \\
\text { G.B., Prithvi Raj, P. }\end{array}$ & 2014 \\
\hline
\end{tabular}

NOTA 2: Las traducciones de los títulos de los artículos son no oficiales.

\section{Plos One}

El primero archivo que se revisa de la fuente Plos One tiene un enfoque en las condiciones asociadas a ventiladores y a la vigilancia de eventos adversos en enfermedades respiratorias por el uso de ventilación mecánica invasiva (Tabla 4). Se realiza un análisis de la gestión y mantenimiento de los ventiladores usando un análisis multivariable de los riesgos de los ventiladores. Los cuatro factores de riesgo encontrados fueron: ausencia de participación intensiva en el manejo de pacientes ventilados, utilización de presión de conducción alta, desarrollo de edema y aumento de peso corporal. Estos criterios son clave para el desarrollo de medidas preventivas. [10]

En segunda instancia se analiza un documento en el que se presenta los implantes cardiacos por el alto riesgo que representan y sus complicaciones asociadas con mortalidad (Tabla 4). Los factores de riesgo desde el punto de vista del dispositivo son problemas de batería del dispositivo y entrega incorrecta de la terapia [10].
Tabla 4. Documentos publicados por Plos One

\begin{tabular}{|c|c|c|c|}
\hline $\mathrm{N}^{\circ}$ & Título & Autores & Año \\
\hline 1 & $\begin{array}{l}\text { Association of patient care } \\
\text { with ventilatorassociated } \\
\text { conditions in critically ill } \\
\text { patients: Risk factor analysis } \\
\text { [Asociación de atención } \\
\text { al paciente con afecciones } \\
\text { asociadas a ventilador en } \\
\text { pacientes críticamente } \\
\text { enfermos: Análisis de } \\
\text { factores de riesgo] }\end{array}$ & $\begin{array}{l}\text { Nakahashi, } \\
\text { S., Yamada, T., } \\
\text { Ogura, } \\
\text { T., (...), Suzuki, } \\
\text { K., Imai, H. }\end{array}$ & 2014 \\
\hline 2 & $\begin{array}{l}\text { Recalls of cardiac implants } \\
\text { in the last decade: What } \\
\text { lessons can we learn? } \\
\text { [Recuerdos de implantes } \\
\text { cardiacos en la última } \\
\text { década: ¿Qué lecciones } \\
\text { podemos aprender?] }\end{array}$ & $\begin{array}{l}\text { Zhang, S., Kriza, } \\
\text { C., Schaller, } \\
\text { S., Kolominsky- } \\
\text { Rabas, } \\
\text { P.L. }\end{array}$ & 2014 \\
\hline
\end{tabular}

NOTA 3: Las traducciones de los títulos de los artículos son no oficiales.

A continuación, se presentan en la Tabla 5 los criterios de riesgo encontrados en los artículos analizados, de acuerdo a los enfoques hallados en las revistas.

Tabla 5. Criterios de riesgo

\begin{tabular}{ll}
\hline \multicolumn{1}{c}{ Enfoque } & \multicolumn{1}{c}{ Criterios de riesgo } \\
\hline \multirow{3}{*}{ Normativo } & Probabilidad de ocurrencia de un evento \\
& Frecuencia de incidentes \\
& Población afectada \\
& Nivel de seguridad de la tecnología \\
& Asignación de método adecuado \\
& Consentimiento informado \\
& Tasa de deserción \\
& Cumplimiento aceptable \\
& Duración del dolor \\
& Tratamientos previos \\
& Duración del seguimiento. \\
& Participación insuficiente en el manejo \\
& de pacientes \\
& Mayor presión de conducción \\
& Cambios en el peso corporal \\
& Desarrollo de edema \\
& Problemas de batería \\
& Terapia incorrecta
\end{tabular}

NOTA 5: Las traducciones de los criterios de riesgo son no oficiales.

\section{Discusión}

En la metodología para la vigilancia tecnológica se plantearon 8 ecuaciones de búsqueda con las palabras clave para este artículo y sus respectivas expresiones equivalentes, se encontraron resultados que en ocasiones no fueron satisfactorios por lo que se optó por modificar 
en la ecuación $\mathrm{N}^{\circ} 6$ a "medical device" para que en la base de datos se encontrara la expresión exacta y arrojara unos resultados mayor exactitud.

Las publicaciones analizadas por fuente tenían como área temática de preferencia Medicina, aunque en cada revista los temas fueron abordados de diferente forma. En la revista Bundesgesundheitsblatt Gesundheitsforschung Gesundheitsschutz desde la normativa para dispositivos médicos en Alemania y la evaluación de riesgos para esta tecnología sanitaria.

En la revista Pain Physician se presentaron instrumentos desarrollados, que se basaron en criterios de revisión de la organización Cochrane, para el manejo del dolor. Mientras en la revista Plos One el enfoque se basó en analizar casos particulares de dispositivos médicos y los respectivos riesgos asociados al uso, así como en la identificación de tales riesgos.

\section{CONCLUSIÓN}

Los resultados de la vigilancia tecnológica se presentaron con base en los documentos publicados en las revistas que mayor cantidad de publicaciones realizaron, en total se analizaron 8 documentos de 3 fuentes. En todas se observa la importancia de gestionar los riesgos en la atención sanitaria para dar seguridad a los pacientes en todo el ciclo de vida de los dispositivos médicos.

En las publicaciones de la revista Bundesgesundheitsblatt Gesundheitsforschung Gesundheitsschutz se observó un particular interés por divulgar los marcos regulatorios alemanes para los dispositivos médicos, especialmente para los que se clasifican como de alto riesgo. Este resultado se apoya con el obtenido por País ya que Alemania es el segundo, después de Estados Unidos, con mayor cantidad de documentos publicados.

La evaluación de criterios de riesgo con base en casos de estudio (o basados en evidencia) son una fuente de conocimiento apropiada para estudiar los dispositivos médicos de forma particular, lo anterior se evidencia por las publicaciones analizadas en las revistas Pain Physician y Plos One.

\section{Agradecimiento}

Los autores agradecen al Programa de Vigilancia Tecnológica e Inteligencia Competitiva de la Universidad Pontificia Bolivariana, por los aportes e indicaciones para la presentación de la información encontrada en la base de datos Scopus.

\section{REFERENCIAS}

[1]. Universidad de Chile, "Vigilando las fronteras tecnológicas." p. 19, 2011.

[2]. L. Tamayo, B. Arango, and A. Fadul, "Vigilancia Tecnológica: Metodologías y Aplicaciones," 2012.

[3]. Dirección de Medicamentos y Tecnologías en Salud, "Evaluación y Gestión de Equipo Biomédico,” 2013.

[4]. H. Acevedo, "Recomendaciones sobre la adquisición de tecnología médica,” 2009. [Online]. Available: http://www. elhospital.com/temas/Recomendaciones-sobre-laadquisicion-detecnologia-medica+8071597. [Accessed: 28-Sep2015].

[5]. ISO, "ISO 31000:2009(en), Risk management - Principles and guidelines," 2009. [Online]. Available: https://www.iso.org/obp/ ui/\#iso:std:iso:31000:ed-1:v1:en.

[6]. UNESCO, "SKOS Criterio de evaluación." [Online]. Available: http://skos.um.es/unescothes/C01445/html.

[7]. ISO, "ISO 55000:2014 - Asset management -- Overview, principles and terminology," 2014. [Online]. Available: http:// www.iso.org/iso/catalogue_detail?csnumber $=55088$.

[8]. UNESCO, "SKOS Equipo Sanitario." [Online]. Available: http:// skos.um.es/unescothes/C02456/html.

[9]. Elsevier, "Scopus." [Online]. Available: http://www.americalatina. elsevier.com/corporate/es/scopus.php.

[10]. Scopus, "Scopus. Risk assessment criteria and 'medical device' management.," 2017. .

[11]. J. D. Estep et al., "Risk assessment and comparative effectiveness of left ventricular assist device and medical management in ambulatory heart failure patients results from the ROADMAP Study," J. Am. Coll. Cardiol., vol. 66, no. 16, pp. 1747-1761, 2015.

[12]. H. Mahfoud, A. El Barkany, and A. El Biyaali, "A hybrid decision-making model for maintenance prioritization in health care systems," Am. J. Appl. Sci., vol. 13, no. 4, pp. 439-450, 2016.

[13]. S. Zhang, C. Kriza, S. Schaller, and P. L. Kolominsky-Rabas, "Recalls of cardiac implants in the last decade: What lessons can we learn?," PLoS One, vol. 10, no. 5, pp. 1-15, 2015.

[14]. S. Nakahashi, T. Yamada, T. Ogura, K. Nakajima, K. Suzuki, and H. Imai, "Association of patient care with ventilator-associated conditions in critically ill patients: Risk factor analysis," PLoS One, vol. 11, no. 4, pp. 1-13, 2016. 